\title{
BMJ Open Observational non-randomised controlled evaluation of the effectiveness of cancer counselling centres: a study protocol
}

\author{
Solveigh Paola Lingens (D) , ${ }^{1}$ Georgia Schilling, ${ }^{2}$ Julia Harms, ${ }^{3}$ Holger Schulz, ${ }^{1}$ \\ Christiane Bleich ${ }^{1}$
}

To cite: Lingens SP, Schilling G, Harms J, et al. Observational non-randomised controlled evaluation of the effectiveness of cancer counselling centres: a study protocol. BMJ Open 2019;9:e032889. doi:10.1136/ bmjopen-2019-032889

- Prepublication history for this paper is available online To view these files, please visit the journal online (http://dx.doi org/10.1136/bmjopen-2019032889).

Received 10 July 2019 Revised 26 October 2019 Accepted 07 November 2019

Check for updates

(C) Author(s) (or their employer(s)) 2019. Re-use permitted under CC BY-NC. No commercial re-use. See rights and permissions. Published by BMJ.

${ }^{1}$ Department of Medical Psychology, University Medical Center Hamburg-Eppendorf, Hamburg, Germany

${ }^{2}$ Oncology, Asklepios Hospital Altona, Hamburg, Germany ${ }^{3}$ Department of Psychology, University of Osnabruck, Osnabruck, Germany

Correspondence to

Ms Solveigh Paola Lingens;

s.lingens@uke.de

\section{ABSTRACT}

Introduction In recent years, medical treatment for cancer has improved, thereby increasing the life expectancy of patients with cancer. Hence, the focus in healthcare shifted towards analysing treatments that offer to decrease distress and improve the quality of life of patients with cancer. The psychological burden of patients with cancer originates from all kinds of psychosocial challenges related to diagnosis and treatment. Cancer counselling centres (CCounCs) try to address these concerns. However, the current literature lacks research on the effectiveness of CCounCs. This study aims to assess the effectiveness of CCounCs with regard to distress and other relevant psychosocial variables (quality of life, anxiety and so on).

Methods and analysis This prospective observational study with a non-randomised control group has three measurement points: before the first counselling session (baseline, t0) and at 2 weeks and 3 months after baseline (t1, t2). Patients and their relatives who seek counselling between December 2018 and November 2020 and have sufficient German language skills will be included. The control group will be recruited at clinics and oncological outpatient centres in Hamburg. Propensity scoring will be applied to adjust for differences between the control and intervention groups at baseline. Sociodemographic data, medical data and counselling concerns are measured at baseline. Distress (distress thermometer), quality of life (Short Form-8 Health Survey, European Organization for Research and Treatment of Cancer Core Quality of Life Questionnaire-Core 30), anxiety (Generalized Anxiety Disorder-7), depression (Patient Health Questionnaire-9) and further psychosocial variables are assessed at all time points. With a total of 787 participants, differences between the intervention and control groups of a small effect size $(f=0.10)$ can be detected with a power of $80 \%$.

Ethics and dissemination The study was registered prior to data collection with the German Registration of Clinical Trials in September 2018. Ethical approval was received by the local psychological ethical committee of the Center for Psychosocial Medicine at the University Medical Centre Hamburg-Eppendorf in August 2018. The results will be published in peer-reviewed journals.

Trial registration number DRKS00015516; Pre-results.
Strengths and limitations of this study

This study is one of few prospective controlled evaluations of cancer counselling centres worldwide.

- The study includes valid and reliable assessment measures.

- The study shows high ecological validity as it seeks to assess a service that has grown out of the community and has contextual relevance to the community.

- Barriers to approaching the services are also assessed.

- Relatives of patients with cancer are often underrepresented or disregarded in research, and since a substantial number of relatives may also seek support at cancer counselling centres they are included in this study.

- Due to ethical considerations, the control group cannot be randomised; however, possible differences in confounding factors at baseline are corrected with the statistical technique of propensity scoring.

\section{INTRODUCTION}

Cancer does not solely affect physical health. A cancer diagnosis and its treatment influence the psychological well-being of the person diagnosed and may also have an effect on the relatives' well-being. ${ }^{1-5}$ Moreover, evidence exists that a cancer diagnosis can also lead to occupational and financial hazards. ${ }^{6-8}$

In the inpatient setting, the treatment of physical issues is usually the main focus. Some clinics offer additional psychological support. ${ }^{910}$ However, as soon as patients are discharged from the hospital, they and their relatives are largely left alone with regard to seeking help. ${ }^{11}$ Psychological support is either available at outpatient care centres or with registered psychotherapists. However, the distress caused by the cancer diagnosis is not necessarily an indicator for psychotherapy, ${ }^{13}$ which is the only psychological care service covered by health insurances in 
Germany. Furthermore, access to psychosocial or legal support is often expensive and not specifically adapted for the situation of a patient with cancer. ${ }^{12}$ In Germany, the German Cancer Society (Deutsche Krebsgesellschaft) is trying to fill the psychosocial care gap with the help of cancer counselling centres (CCounCs). ${ }^{14}$

According to a current inventory and analysis of psychooncological care in Germany, there are approximately 160 CCounCs in Germany; between centre visits and home visits, each centre cares for on average 338 patients and relatives per year. ${ }^{15}$ The services of the CCounCs include psychological, social and legal support for patients with cancer and their relatives, as well as the provision of medical information. The counselling can be sought out as often as needed, free of charge. The staff consists of social workers, social pedagogues, psycho-oncologists and medical doctors. In addition to individual counselling, the CCounCs offer a variety of workshops and courses for patients with cancer and their relatives. Unfortunately, although found to be useful by most patients with cancer visiting the centres, these services are not covered by health insurance. ${ }^{16}$ Financing depends on charity funds and is therefore limited. There are 119 CCounCs in Germany that are funded by the German Cancer Society (Deutsche Krebsgesellschaft). However, most of them have no more than four counsellors. Other countries have established similar services with similar limitations. ${ }^{17}$

\section{Evidence gap}

As with other interventions designed to improve the well-being of patients, the effectiveness of such interventions needs to be verified in high-quality scientific studies before health insurance companies will decide to cover them. However, psychological interventions do not solely decrease the psychological distress experienced by patients with cancer and their relatives. Low-threshold psychological and psychosocial support ought to be of major importance to help patients with cancer and their families with everyday challenges and has the benefit of reaching a larger group of people with different needs. ${ }^{18} 19$ The first non-systematic literature search revealed that most studies that evaluated CCounC services were post-hoc cross-sectional studies. One German study retrospectively evaluated the services and quality of 26 CCounCs in Germany. ${ }^{20}$ They found that the majority of the participants were satisfied with the services. Unfortunately, current research lacks prospective longitudinal studies that include a control group and assess the effectiveness of CCounCs.

To assess the effectiveness of cancer counselling services, outcome measures were chosen according to their prevalence in patients with cancer and positive response to psychosocial interventions. Because distress was found to be highly prevalent in patients with cancer ${ }^{21} 22$ and responds well to psychosocial interventions, ${ }^{2324}$ this study aims to assess the effectiveness of CCounCs primarily with regard to distress. In a secondary step, the effectiveness will be assessed with regard to measures such as quality of life, sense of mastery, anxiety, fatigue and depression because those measures showed improvement after psychosocial interventions. ${ }^{19}{ }^{2325-27}$ To gain more insights into factors that may influence patient satisfaction with the counselling, medical and sociodemographic variables as well as satisfaction with the counselling and with the counsellor will be assessed. The needs for, requests for and utilisation of psychosocial counselling at the CCounCs will also be evaluated. Furthermore, the possible barriers that prevent patients or relatives from approaching CCounCs will be determined. Moreover, a comprehensive assessment of possible predictors of an effective counselling session will be conducted. A counselling session is considered to be effective if the outcome measure (distress) improves after the session. Hence, the main research hypotheses are as follows:

1. Patients who make use of CCounC services will show a greater improvement in the primary outcome (distress) compared with the control group.

2. Patients who make use of CCounC services will show greater improvement in the secondary outcomes compared with the control group.

Further research questions are as follows:

1. What factors (ie, sociodemographic, medical, counsellor-patient alliance) predict satisfaction with the services and higher primary and secondary outcome scores?

2. What are the needs and requests for cancer counselling services among patients with cancer and their relatives?

3. What are possible barriers to the utilisation of CCounC services by patients with cancer and their relatives?

\section{METHODS AND ANALYSIS}

\section{Study design}

To test the hypotheses and address the research questions, a quasi-experimental prospective study will be conducted. To obtain largely valid results, a non-randomised control group will be included, which will be statistically matched with the intervention group at baseline. The CCounCs are represented by two CCounCs in Hamburg, Germany. To measure the possible effects of individual counselling sessions at the CCounCs, the assessment will be carried out at three measurement time points to capture shortterm as well as mid-term effects. The baseline measure will be applied immediately before the first counselling session. The postmeasure will follow 2 weeks after the first session to avoid carry-over effects. The follow-up will be distributed 3 months after baseline. The measurement time points for the control group are comparable with the intervention group.

\section{Measurement time points}

Intervention group (patients with cancer and relatives seeking advice at CCounCs).

- Baseline (before the first counselling session).

- Follow-up: 2 weeks after baseline. 
- Follow-up: 12 weeks after baseline.

Control group (patients with cancer and relatives).

- Baseline.

- Follow-up: 2 weeks after baseline.

- Follow-up: 12 weeks after baseline.

For an overview of measures at each time point, see table 1 .

\section{Individual counselling (intervention group)}

The intervention group will be recruited at the two CCounCs of the Cancer Society Hamburg in HamburgEppendorf and Hamburg-Harburg. The CCounCs offer mainly psychological and social/legal support in the form of individual counselling sessions. The type of support can be combined and depends on patients' requests. Medical counselling is only offered in the form of general information on treatment options or alternatives. Workshops and courses are offered but will not be evaluated in this study due to the small participant pool and for cost-benefit reasons. The CCounCs will be approached by patients and relatives at any time after a cancer diagnosis and throughout the course of the disease or after achieving a remission. In this study, at least one individual counselling session will be considered an intervention.

\section{Cooperation partners (control group)}

The control group recruitment will be carried out in cooperation with several outpatient centres and clinics in Hamburg. To address possible regional differences, the two counselling centres will be compared with cooperating clinics in the same region. The cooperating partners that will be approached are the Asklepios Clinic in Altona, Hamburg; the Haematological and Oncological Outpatient Centre (HOPA) in Altona, Hamburg; Dr Mattner, Gynaecological Practice Clinic, Hamburg; Oncological Centre, Marienhospital, Hamburg; Dr Verpoort and Dr Wierecky, specialised practice for oncology and haematology, Hamburg; Dr Faak, HaematologyOncology, Hamburg; Asklepios Clinic Harburg, Hamburg; Helios Mariahilf Clinic, Hamburg; Professor Laack, Haematology-Oncology, Hamburg; and Dr Mohr, Oncology Lerchenfeld, Hamburg.

\section{Inclusion and exclusion criteria}

For the intervention group, patients with cancer and their relatives (>18 years) who contact a CCounC for the first time during the recruitment phase will be included. All subjects will have to sign an informed consent form to

Table 1 Outcome measures, instruments and time of assessment

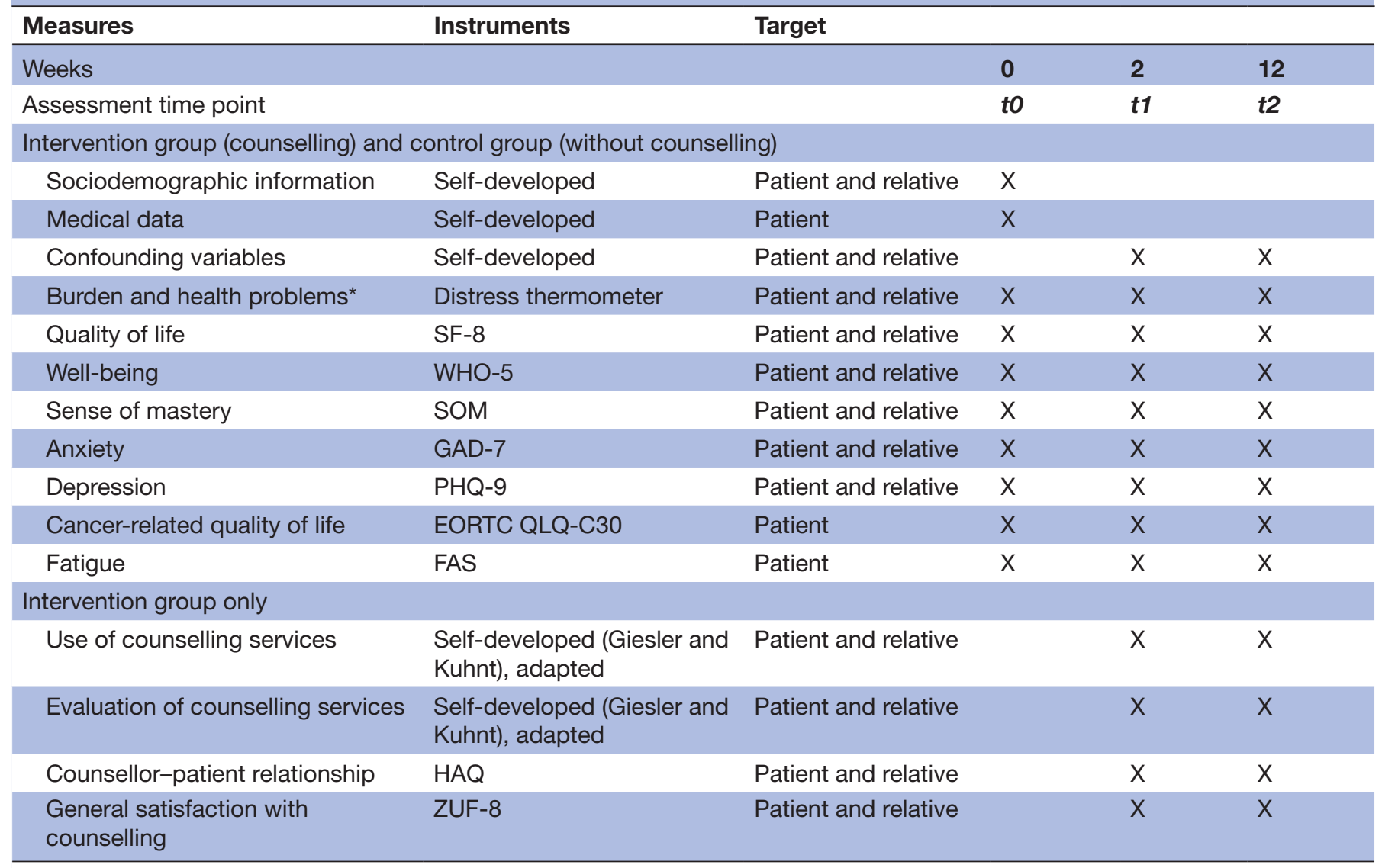

*Primary outcome.

EORTC QLQ-C30, European Organization for Research and Treatment of Cancer Quality of Life Questionnaire-Core 30; FAS, Fatigue Assessment Scale; GAD-7, Generalized Anxiety Disorder; HAQ, Helping Alliance Questionnaire; PHQ-9, Patient Health Questionnaire; SF-8, Short Form-8 Health Survey; SOM, Sense of Mastery Scale; ZUF-8, Patient Satisfaction Questionnaire. 
participate. We will exclude patients and relatives who do not have sufficient German language skills to complete the questionnaire and who have severe physical or mental constraints. Patients and relatives with cognitive limitations will also be excluded.

For the control group, patients with cancer and their relatives who seek help at CCounCs at baseline or during the data collection will be excluded from the control group and included in the intervention group with a new baseline measure. Patients who are visiting a psychologist or psycho-oncologist during data collection will be excluded from the actual control group and will be assigned to a separate control group, which will be analysed and compared with the intervention group separately.

\section{Recruitment and procedure}

\section{Intervention group}

Patients and relatives who seek advice in one of the two CCounCs in Hamburg from December 2018 to November 2020 will be approached. Before their first individual counselling session, they will receive oral and written information on the aims and procedure of the study and will be asked to participate. If they agree to take part in the study, they will give their written consent to participate and provide their general contact data (name, address, phone number). Subsequently, they will receive the baseline questionnaire and will be given sufficient time to complete it before the counselling session starts. The contact information will be transferred to the master key. The master key is a printed overview of participants' identification codes for the study and their corresponding contact data, which will be used to match the baseline questionnaires with the follow-up data. After the participants have completed the baseline questionnaire, the master key, consent and questionnaire will be securely stored at the Department of Medical Psychology (University Medical Center Hamburg-Eppendorf). The two follow-up questionnaires will be sent by mail after 2 weeks and after 12 weeks. To record at what time participants have actually filled in the questionnaire, they will be asked to report the date at the beginning of the questionnaire. The identification code will be placed on both follow-up questionnaires to match the data. A personalised letter and a stamped return envelope will be enclosed with the respective questionnaire. Access to the data will only be granted to the scientific staff at the Department of Medical Psychology.

\section{Control group}

Patients with cancer and their relatives will be approached in the waiting rooms or in their stationary rooms of the corresponding clinic or outpatient centre. The potential participants will receive oral and written information on the aims and procedure of the study. If they agree to take part in the study, they will give their written consent to participate and provide their general contact data (name, address, phone number). Subsequently, they will receive the baseline questionnaire. After the participants completed the baseline questionnaire, the contact data, consent and questionnaire will be securely stored at the Department of Medical Psychology (University Medical Center Hamburg-Eppendorf). The two follow-up questionnaires will be sent by mail after 2 weeks and after 12 weeks. To record at what time participants have actually filled in the questionnaire, they will be asked to report the date at the beginning of the questionnaire. The identification code will be placed on both follow-up questionnaires to match the data. A personalised letter and a stamped return envelope are enclosed with the respective questionnaire. Access to the data will only be granted to the scientific staff at the Department of Medical Psychology. If participants seek advice after the administration of the baseline measure, they will be reassigned to the intervention group.

For both groups, no incentives will be offered for participation. Participants who will not return the questionnaires within 2 weeks will receive a reminder by post. Blinding participants or researchers is not possible due to the design of the study.

For a detailed overview of the procedure and study arms, see figure 1 .

\section{Outcome and measurements}

Distress thermometer (primary outcome)

The distress thermometer has been found to be sensitive for measuring distress in patients with cancer. ${ }^{28}$ It consists of a general distress score from 0 to 10 , where 10 indicates high distress. For values above the cut-off $(\geq 5)$, it is assumed that there is a need for psychological support. In addition, the distress measure encompasses a detailed list of 35 problems that may provide an explanation for elevated levels of distress. The list groups the problems in categories such as practical, family, emotional, physical and spiritual concerns. Participants can indicate whether any of the problems applies to them by ticking Yes or No. The German version of the measure has been validated and is often used in clinical practice as a screening tool. ${ }^{29}$

\section{Quality of life}

The Short Form-8 Health Survey (SF-8) survey is the short and second version of the SF-36. ${ }^{30-32}$ It measures healthrelated quality of life. Contrary to the developed SF-12, ${ }^{33}$ which assesses the two main dimensions of physical and mental health, the SF-8 encompasses all eight dimensions of the original SF-36. The SF-8 has been found to be a valid and reliable questionnaire. For this study, the German version will be used. ${ }^{34}$ The evaluation is equivalent to that of the SF-36. ${ }^{30}$

A cancer-specific quality of life measure is the European Organization for Research and Treatment of Cancer Quality of Life Questionnaire-Core 30 (EORTC QLQ-C30). ${ }^{35}$ The questionnaire consists of 30 items with over 10 different subscales. The questionnaire has been found to be valid and reliable for most cancer diagnoses and has been translated into over 100 languages. ${ }^{36} 37$ The subscales assess physical functioning, role function, 


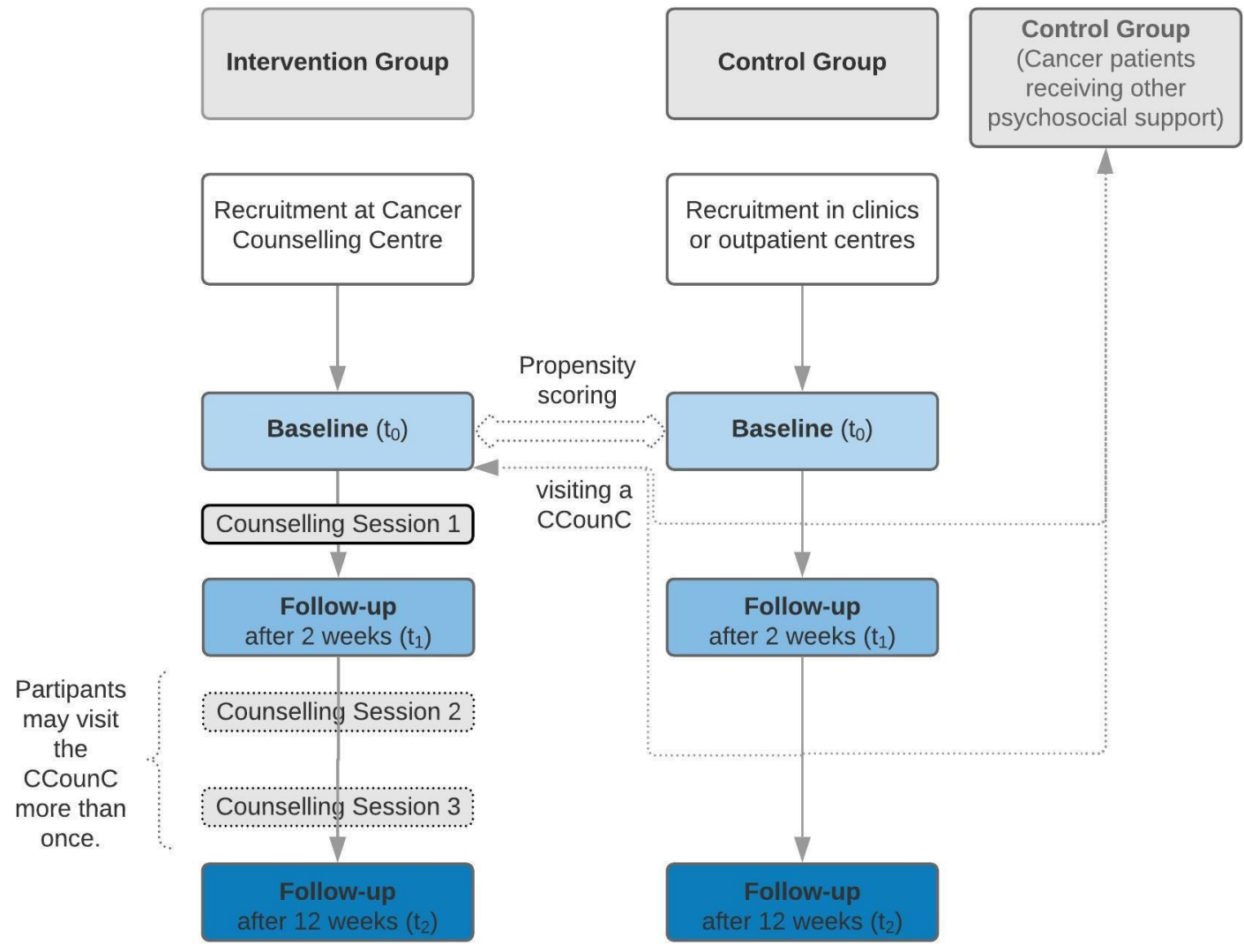

Figure 1 Study procedure for the intervention and control groups. CCounC, cancer counselling centre.

cognitive and emotional function, pain, other physical symptoms related to cancer, and global health status.

\section{Well-being}

The WHO developed a short rating scale to measure subjective and generic well-being. ${ }^{38}$ The WHO-5 is a fiveitem scale that has received international acceptance and has been suggested to be valid as a depression screening tool as well as an outcome measure in clinical trials. The scores range from 0 (none of the time) to 5 (all of the time). Higher scores indicate greater well-being. The psychometric properties of the German version of the WHO-5 have been found to be excellent. ${ }^{39}$

\section{Depression}

The Patient Health Questionnaire (PHQ-9) was originally developed and tested in a large study including over 6000 patients. Because the original measure included 27 items assessing a variety of health aspects, it was shortened and separated into individual questionnaires such as the PHQ-9 among others. ${ }^{40}{ }^{41}$ It proved efficient in assessing and monitoring depression severity in different settings. Items are scored from 0 (not at all) to 3 (nearly every day), with higher scores demonstrating more serious symptoms of depression.

\section{Anxiety}

The Generalized Anxiety Disorder (GAD-7) questionnaire was developed from the same questionnaire as the PHQ-9. The scoring of the GAD-7 is thus the same as for the PHQ-9. It is widely used to screen for anxiety, especially social anxiety, panic and post-traumatic stress disorder. $^{42}$

\section{Fatigue}

In addition to anxiety and depression, fatigue is one of the most common symptoms in patients with cancer. However, symptoms are often confused with depressive symptoms. The Fatigue Assessment Scale assesses fatigue and distinguishes it from related concepts. ${ }^{43}$ The psychometric properties of the questionnaire have been found to be good across different studies. ${ }^{44}$ The total score differentiates between no fatigue and substantial fatigue and ranges from 10 to 50 .

\section{Sense of mastery}

The Pearlin's Sense of Mastery Scale assesses personal control, which combines aspects of perceived self-efficacy and the construct of the internal locus of control. It was originally developed by Pearlin and Schooler. ${ }^{45}$ The original version includes seven items such as 'I have little control over the things that happen to me'. The short version of four items was first tested by Badura et $a l$. in $1987 .{ }^{46}$ All items are negatively worded and require reverse coding prior to scoring. The response options were offered on a 4-point Likert scale. The score ranges from 4 to 16, with higher scores indicating a greater level of mastery. The questionnaire meets the test quality 
criteria $^{47}$ and correlates with several scales measuring well-being and depression. ${ }^{48}$

\section{Satisfaction}

The Patient Satisfaction Questionnaire (ZUF-8) is the eight-item German version of the Client Satisfaction Questionnaire- $8 .{ }^{49}$ It has been found to be valid in measuring patient satisfaction. The reliability has also been found to be generally high in both the original ${ }^{49}$ and the German version..$^{50}$ Previous assessments show a tendency towards high satisfaction resulting in a left-skewed distribution, which needs to be considered in the evaluation of the results.

\section{Counsellor-patient relationship}

The Helping Alliance Questionnaire (HAQ) was originally developed to assess the relationship between the therapist and the patient in a short-term therapy setting. ${ }^{51}$ The questionnaire was translated into German by Bassler and colleagues. ${ }^{52}$ Validity and reliability are sufficiently high for the 11-item questionnaire ${ }^{5253}$ For this study, the wording of the HAQ items was slightly adapted to match the context of the relationship assessed. Hence, 'therapist' was replaced by 'counsellor'. Furthermore, two items that did not reflect the context of a counselling relationship were excluded.

\section{Evaluation and use of counselling services}

To evaluate the expectation and use of counselling services, a specifically developed questionnaire by Giesler and Kuhnt is used, ${ }^{20}$ which has been shown useful for assessing and evaluating relevant aspects of counselling requests. For this study, the questionnaire was adjusted to obtain a more comprehensive impression of the use of counselling services. Therefore, item 7 , which assesses the general support to deal with the altered life situation, was split into three items assessing the wish for support in dealing with the health, financial and occupational life situation.

\section{Confounding variables}

The following factors will be controlled for by including them in the analyses. For the intervention group, the number of counselling visits will be adjusted for. It will also be assessed whether participants in the control group attend counselling sessions during the course of the study. If participants of the control group attend a counselling session during the study, they will be assigned to the intervention group. Possible differences between the control and intervention groups will be adjusted for the following confounding variables to minimise bias: time since diagnosis, cancer type and status, cancer treatment, metastasis, other diseases (eg, cardiovascular diseases), age, sex, relationship status, socioeconomic background, current occupational status, educational background, and financial situation. Additionally, previous contact with counselling centres (before the beginning of the study), medication (influencing the mood) and contact with psychological staff (psychiatrist, psychologist and so on) before or during the study will also be controlled for.
Furthermore, to address possible regional differences, the counselling centre in Hamburg-Eppendorf will be compared with two clinics in the same region (HOPA and Asklepios Clinic). The counselling centre in HamburgHarburg will be compared with two clinics in Harburg (Gynaecological Praxis and Mariahilf Clinic in Harburg).

\section{Patient and public involvement}

Patients and members of the public were involved at several stages during the development of the study protocol. In a pilot study, patients gave feedback on the first draft of the questionnaire. Their opinions on the length, design and relevance of the questions and content were considered for the final version. Members of the Cancer Society Hamburg were consulted to assess the possible burden of the study on the patients, to develop the outcome measures and to plan the recruitment and study procedure.

\section{Quantitative analyses}

The sample will be divided into a control group and an intervention group. At first, descriptive analyses will be conducted for baseline and follow-up measures of both groups. To account for the previously mentioned confounding factors, differences between the control and intervention groups at baseline will be adjusted using propensity scoring. To test hypothesis 1 (patients who make use of cancer counselling services will show a greater improvement in the primary outcome (distress) compared with the control group) and hypothesis 2 (patients who make use of cancer counselling services will show greater improvement in the secondary outcomes compared with the control group), a linear mixed models analysis will be performed. To answer the third research question, three linear regressions will be applied. For research questions 2 and 3, frequency analyses will be applied. For all analyses, IBM SPSS Statistics software (V.25 or higher) will be used.

\section{Sample size and power}

The power analysis was based on an analysis of covariance as it is comparable with a mixed models analysis in wide format. To detect significant group differences with a small effect size of $\mathrm{f}=0.10$ and a power of $80 \%$, a total sample size of 787 participants is needed ( $n=394$ for each group). For the intervention group, the CCounCs in Hamburg have estimated that approximately 900 new patients or relatives make use of cancer counselling services each year. With an estimated response rate of $60 \%$, approximately 540 participants can be expected at baseline each year. With a dropout rate of $40 \%$ between each follow-up, approximately 194 participants can be expected at the final follow-up within 1 year. Therefore, the calculated number of participants for the intervention group can be attained with a data collection time frame of 2 years. Due to a high number of cooperating partners, it is presumable considering similar response and dropout rates that 
the expected number of participants in the control group will be reached within 2 years. For the control group, the number of participants will be held fairly equal across recruitment sites.

\section{Ethics and dissemination}

The study was registered prior to data collection with the 'German Registration of Clinical Trials' in September 2018. Participants provided written informed consent before data collection. They are also provided with written information about the study. Since the study is mere observational, no additional intervention is integrated. The intervention is part of the general care offered to patients with cancer in Hamburg.

The study was initiated in April 2018, and the anticipated duration is 3 years. The preparation period lasted until November 2018. The recruitment of the intervention group started in December 2018 at the CCounC in Hamburg-Eppendorf and in January 2019 at the CCounC in Hamburg-Harburg. The data collection period is anticipated to last 2 years; the plan is to complete data collection in December 2020. The recruitment of participants in the control group began in February 2019 with two cooperating partners. The remaining cooperating partners are planned to be included until October 2019. Throughout the data collection, data will be processed and managed continuously. Findings will be published in peer-reviewed journals and presented at national and international conferences and congresses. A report of the results will also be made available for counsellors and clinicians via the German Cancer Society.

Acknowledgements The authors acknowledge the following cooperating partners for their participation in the study: Asklepios Clinic in Altona, Hamburg; the Oncological Outpatient Centre (HOPA) in Altona, Hamburg; Dr Mattner and Dr Großmann, Gynaecological Practice Clinic, Hamburg; and Dr Großmann, Breast Centre, Mariahilf Clinic, Hamburg.

Contributors $\mathrm{CB}$ is the principal investigator of the study. The idea and concept were developed by GS, CB and HS. SPL and JH are responsible for the preparation of study materials and data collection. SPL will analyse the data and is responsible for writing the study protocol. $\mathrm{CB}$ and $\mathrm{HS}$ revised and approved the final manuscript. $\mathrm{CB}, \mathrm{HS}$ and SPL agree to be accountable for the work.

Funding The study is funded by the Cancer Society of Hamburg (Hamburger Krebsgesellschaft). The Cancer Society of Hamburg is not involved in the execution of the study or any data analyses or writing of the manuscript.

Competing interests None declared.

Patient consent for publication Not required.

Ethics approval The study protocol was approved by the local psychological ethical committee of the Center for Psychosocial Medicine (LPEK) at the University Clinic Hamburg-Eppendorf on 22 August 2018 (no: LPEK-007).

Provenance and peer review Not commissioned; externally peer reviewed.

Open access This is an open access article distributed in accordance with the Creative Commons Attribution Non Commercial (CC BY-NC 4.0) license, which permits others to distribute, remix, adapt, build upon this work non-commercially, and license their derivative works on different terms, provided the original work is properly cited, appropriate credit is given, any changes made indicated, and the use is non-commercial. See: http://creativecommons.org/licenses/by-nc/4.0/.

\section{ORCID iD}

Solveigh Paola Lingens http://orcid.org/0000-0003-4230-0389
REFERENCES

1 Hagedoorn M, Sanderman R, Bolks HN, et al. Distress in couples coping with cancer: a meta-analysis and critical review of role and gender effects. Psychol Bull 2008;134:1-30.

2 Asai M, Akechi T, Nakano T, et al. Psychiatric disorders and background characteristics of cancer patients' family members referred to psychiatric consultation service at National cancer center hospitals in Japan. Palliat Support Care 2008;6:225-30.

3 Grunfeld Eet al. Family caregiver burden: results of a longitudinal study of breast cancer patients and their principal caregivers. Can Med Assoc J 2004;170:1795-801.

4 Lederberg MS. The family of the cancer patient. In: Holland J, ed. Psycho-Oncol. New York and Oxford: Oxford University Press, 1998: 981-93.

5 Hodges LJ, Humphris GM, Macfarlane G. A meta-analytic investigation of the relationship between the psychological distress of cancer patients and their carers. Soc Sci Med 2005;60:1-12.

6 Lu L, O'Sullivan E, Sharp L. Cancer-Related financial hardship among head and neck cancer survivors: risk factors and associations with health-related quality of life. Psychooncology 2019;28:863-71.

7 Bernard DSM, Farr SL, Fang Z. National estimates of out-of-pocket health care expenditure burdens among nonelderly adults with cancer: 2001 to 2008. J Clin Oncol 2011;29:2821-6.

8 Lentz R, Benson AB, Kircher S. Financial toxicity in cancer care: prevalence, causes, consequences, and reduction strategies. J Surg Oncol 2019;120:85-92.

9 Singer S, Dieng S, Wesselmann S. Psycho-oncological care in certified cancer centres--a nationwide analysis in Germany. Psychooncology 2013;22:1435-7.

10 Mehnert A, Koch U. Psychosocial care of cancer patients-international differences in definition, healthcare structures, and therapeutic approaches. Support Care Cancer 2005;13:579-88.

11 Boberg EW, Gustafson DH, Hawkins RP, et al. Assessing the unmet information, support and care delivery needs of men with prostate cancer. Patient Educ Couns 2003;49:233-42.

12 Chapman K, Rush K. Patient and family satisfaction with cancerrelated information: a review of the literature. Can Oncol Nurs $J$ 2003;13:107-16.

13 Kornblith AB. Psychosocial adaptation of cancer survivors. In: Holland J, ed. Psycho-Oncol. New York and Oxford: Oxford University Press, 1998.

14 Beckmann MW, Brucker C, Hanf V, et al. Quality assured health care in certified breast centers and improvement of the prognosis of breast cancer patients. Onkologie 2011;34:362-7.

15 Schulz H, Bleich C, Dabs M, et al. Psychoonkologische Versorgung in Deutschland: Bundesweite Bestandsaufnahme und analyse: Bundesministerium für Gesundheit 2018.

16 e.V. DK. Positionspapier Der Deutschen Krebshilfe und Der Deutschen Krebsgesellschaft Zur Weiterentwicklung und Sicherung Der ambulanten psychosozialen Krebsberatungsstellen in Deutschland 2012.

17 Eakin EG, Strycker LA. Awareness and barriers to use of cancer support and information resources by $\mathrm{HMO}$ patients with breast, prostate, or colon cancer: patient and provider perspectives. Psychooncology 2001;10:103-13.

18 Boltong A, Ledwick M, Babb K, et al. Exploring the rationale, experience and impact of using cancer information and support (cis) services: an international qualitative study. Supportive Care in Cancer 2017;25:1221-8.

19 Gordon LG, Beesley VL, Scuffham PA. Evidence on the economic value of psychosocial interventions to alleviate anxiety and depression among cancer survivors: a systematic review. Asia Pac J Clin Oncol 2011;7:96-105.

20 Ernst J, Eichhorn S, Kuhnt S, et al. [Outpatient psychosocial counseling--results of a user-based cross-sectional study about patient reported needs and its consideration]. Psychother Psychosom Med Psychol 2014;64:421-30.

21 Carlson LE, Zelinski EL, Toivonen KI, et al. Prevalence of psychosocial distress in cancer patients across 55 North American cancer centers. J Psychosoc Oncol 2019;37:5-21.

22 Mehnert A, Hartung TJ, Friedrich $M$, et al. One in two cancer patients is significantly distressed: prevalence and indicators of distress. Psychooncology 2018;27:75-82.

23 Cousson-Géalie F, Bruchon-Schweitzer M, Atzeni T, et al. Evaluation of a psychosocial intervention on social support perceived control, coping strategies, emotional distress, and quality of life of breast cancer patients. Psychol Rep 2011;108:923-42.

24 Schneider S, Moyer A, Knapp-Oliver S, et al. Pre-intervention distress moderates the efficacy of psychosocial treatment for cancer patients: a meta-analysis. J Behav Med 2010;33:1-14. 
25 Goedendorp MM, Gielissen MFM, Verhagen CA, et al. Psychosocial interventions for reducing fatigue during cancer treatment in adults. Cochrane Database Syst Rev 2009;110.

26 Mehnert A, Koch U. Psychological comorbidity and health-related quality of life and its association with awareness, utilization, and need for psychosocial support in a cancer register-based sample of longterm breast cancer survivors. J Psychosom Res 2008;64:383-91.

27 Osborn RL, Demoncada AC, Feuerstein M. Psychosocial interventions for depression, anxiety, and quality of life in cancer survivors: meta-analyses. Int J Psychiatry Med 2006;36:13-34.

28 Holland JC, Bultz BD. The NCCN guideline for distress management: a case for making distress the sixth vital sign. J Natl Compr Canc Netw 2007;5:3-7.

29 Mehnert A, Muller D, Lehmann C, et al. The German version of the NCCN distress thermometer: validation of a screening instrument for assessment of psychosocial distress in cancer patients. Z Psychiatr Psych Ps 2006;54:213-23.

30 Ware JE, Kosinski M, Dewey JE, et al. How to score and interpret single-item health status measures: a manual for users of the SF-8 health survey. Boston: QualyMetric, 2001.

31 Ware JE, Kosinski M, Dewey JE. How to score version 2 of the SF-36 health survey (standard \& acute forms. 3 edn. Lincoln, RI: QualityMetric, 2001.

32 Ware JE. Sf-36 health survey: manual and interpretation guide. Boston, Massachusetts: The Health Institut, New England Medical Center, 1993

33 Ware JE, Kosinski M, Keller SD. A 12-item short-form health survey Construction of scales and preliminary tests of reliability and validity. Med Care 1996;34:220-33.

34 Beierlein V, Morfeld M, Bergelt C, et al. Messung Der gesundheitsbezogenen Lebensqualität MIT dem SF-8. Diagnostica 2012;58:145-53.

35 Fayers PM, Bjordal K, Groenvold M, et al. The EORTC QLQ-C30 scoring manual. Brussels: European Organization for Research and Treatment of Cancer, 2001.

36 Hjermstad MJ, Fossa SD, Bjordal K, et al. Test/retest study of the European organization for research and treatment of cancer core quality-of-life questionnaire. JCO 1995;13:1249-54.

37 Niezgoda HE, Pater JL. A validation study of the domains of the core EORTC quality of life questionnaire. Qual Life Res 1993;2:319-25.

38 Staehr Johansen K. The use of well-being measures in primary health care - the Dep-care project; in World Health organization, regional office for Europe: well-being measures in primary health care - the DepCare project. Geneva: World Health Organization, 1998: E60246.

39 Brähler E, Muehlan H, Albani C, et al. Testing and standardization of the German version of the EUROHIS-QOL and WHO-5 quality-of life-indices. Diagnostica 2007;53:83-96.
40 Spitzer RL, Kroenke K, Williams JB. Validation and utility of a selfreport version of PRIME-MD: the PHQ primary care study. primary care evaluation of mental disorders. patient health questionnaire. JAMA 1999;282:1737-44.

41 Spitzer RL, Williams JBW, Kroenke K, et al. Validity and utility of the PRIME-MD patient health questionnaire in assessment of 3000 obstetric-gynecologic patients: the PRIME-MD patient health questionnaire obstetrics-gynecology study. Am J Obstet Gynecol 2000;183:759-69.

42 Spitzer RLet al. Utility of a new procedure for diagnosing mental disorders in primary care. JAMA 1994;272:1749-56.

43 Michielsen HJ, De Vries J, Van Heck GL. Psychometric qualities of a brief self-rated fatigue measure: the fatigue assessment scale. $J$ Psychosom Res 2003;54:345-52.

44 de Vries J, van der Steeg AF, Roukema JA. Psychometric properties of the fatigue assessment scale (Fas) in women with breast problems. J Health Psychol 2010;10:125-39.

45 Pearlin LI, Schooler C. The structure of coping. J Health Soc Behav 1978;19:2.

46 Badura B, Kaufhold G, Lehmann H, et al. Leben MIT dem Herzinfarkt Eine sozialepidemiologische studie. Berlin, Heidelberg, New York: Springer, 1987.

47 Rüesch P, Bartlomé P, Huber C. Evaluation von Messinstrumenten für den strategischen Bereich »psychische Gesundheit/Stress". Winterthur: Zürcher Hochschule für Angewandte Wissenschaften [ZHAW], 2006.

48 Rüesch P, Manzoni P. Psychische Gesundheit in Der Schweiz: monitoring. Neuchâtel: Schweizerisches Gesundheitsobservatorium, 2003

49 Atkisson CC, Zwick R. The client satisfaction Questionnaire. Psychometric properties and correlations with service utilization and psychotherapy outcome. Eval Program Plann 1983;5:233-7.

50 Schmidt J, Lamprecht F, Wittmann WW. Zufriedenheit MIT Der stationären Versorgung. Entwicklung eines Fragebogens und erste Validitätsuntersuchungen. Psychother Psychosom Med Psychol 1989;39:248-55

51 Alexander LB, Luborsky L. The Penn Helping Alliance Scales. In: Greenberg LS, Pinsof W, eds. The psychotherapeutic process: a research Handbook. New York: Guilford, 1986: 325-66.

52 Bassler M, Potratz B, Krauthauser H, et al. HAQ) von Luborsky. Möglichkeiten zur Evaluation des therapeutischen Prozesses von stationärer Psychotherapie [The "Helping Alliance Questionnaire" (HAQ) by Luborsky]. Psychotherapeut 1995;40:23-32.

53 Bassler M, Nübling R. Helping Alliance Questionnaire. In: G K, S B, B E, eds. Diagnostische Verfahren in Der Psychotherapie. 3th edn. Göttingen: Hogrefe Verlag, 2015: 246-9. 